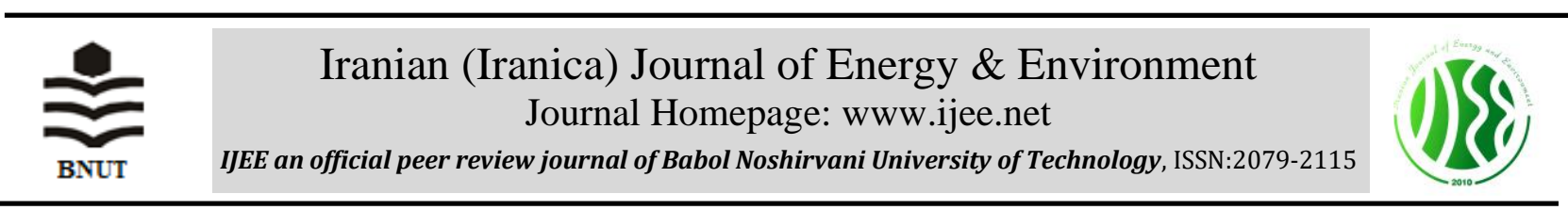

\title{
Deep Learning Based Electricity Demand Forecasting in Different Domains
}

\author{
M. Imani* \\ Faculty of Electrical and Computer Engineering, Tarbiat Modares University, Tehran, Iran
}

\section{$P A P E R \quad I N F O$}

\section{Paper history:}

Received 27 November 2019

Accepted in revised form 02 February 2020

\section{Keywords:}

Frequency Domain

Load Forecasting

Long-Short Term Memory

Time Domain

\begin{abstract}
$A B S T R A C T$
Electricity demand forecasting is an important task in power grids. Most of researches on electrical load forecasting have been done in the time domain. But, the electrical time series has a non-stationary inherence that makes hard load prediction. Moreover, valuable information is hidden in the electrical load sequence which is not open in the time domain. To deal with these difficulties, a new electricity demand forecasting framework is proposed in this work. In the proposed framework, at first, a new feature space of electrical load sequence is composed. The provided domain involves complementary information about shape and variations of electrical load sequence. Then, the obtained load features are integrated with the original load values in time domain to allow a rich input for predictor. Finally, a powerful deep learning technique from the family of recurrent neural networks, named long-short term memory, is used to learn electricity demand from the provided features in single and hybrid domains. The following domains are investigated in this work: frequency, cepstrum, spectral centroid, spectral roll-off, spectral flux, energy, time difference, frequency difference, Gabor and collaborative representation. The experiments show that the use of time difference domain decreases the mean absolute percent error from 0.0332 to 0.0056 .
\end{abstract}

doi: $10.5829 /$ ijee.2020.11.01.06

\section{INTRODUCTION}

An accurate electrical load prediction is necessary to build an intelligent energy management system, adjust and monitor energy demand and supply. It plays an important and crucial role in the present and future energy market [1,2]. All of forecasting types, grouped based on time intervals, such as short-term, medium-term and long-term are serious subjects for planning and operation of electricity industry [3-6]. Shortterm load forecasting (STLF), which is focused in this work, refers to load prediction from several minutes or several hours to several days or a week. Energy providers and utilities need STLF to specify the accurate required amount of electrical energy to purchase. In this way, they can buy electricity with lower prices. Advanced metering infrastructure assemble much more information by providing smart meter data than the data provided by traditional meters. This information allows a potential for an accurate STLF.

Analysis of the load data can be done through several main approaches: deterministic, statistics and artificial intelligence. Almost in all methods, the load sequence is considered as a signal or time series. The deterministic approaches model the relation between consumed (or demand) load with other related factors like weather conditions such as temperature. They do forecasting using curve fitting, smoothing methods and data extrapolation [7-9]. The statistical approaches consider the load time series as a stochastic process. They modelled the load curve of customers in different conditions by using probabilistic approaches such as Bayesian framework [10], regression methods such as autoregressive integrated moving average (ARIMA) [11, 12], support vector regression [13, 14] and Kalman filtering [15]. The artificial intelligence approaches are divided into two main groups: expert systems such as fuzzy based decision makers [16, 17] and artificial neural network (ANNs) [18-20]. The expert systems utilize a knowledge base provided by experts of electricity industry and inference engines constructed based on fuzzy logic. ANNs are known as powerful tools for load forecasting. They are inspired by biological structure of human brain that have several excellent characteristics. They can extract a non-linear model of observations without any assumption about the statistical distribution of data. So, ANNs have capability of dealing with complex patterns opposed to other traditional methods such as ARIMA. ANNs are self-adaptive and data-driven where an appropriate model of available samples is formed adaptively based on the observed data.

The multilayer perceptron with a single hidden layer as a feed foreward neural network (FFNN) has been widely used for load forecasting [21]. But, the main disadvantage of FFNN 
is that it only exploits the current samples of data without considering the previous input samples. In other words, it has not memory to remember anything happened in the past. But, in the sequential data such as load sequences, there are samples related to each other located in time order. To deal with these sequences, recurrent neural networks (RRNs) have been introduced where they consider the input samples previously received together with the current input ones [22]. An extended version of RNNs is long-short term memory (LSTM) network [23, 24]. LSTM has longer memory than RNN such that it is appropriate to learn from input samples and what experienced from past time with very long memory.

The load data, as a temporal sequence or time series, contains worthful information about consumption behavior of customers in successive time intervals. This historical data in the time domain has been used for load forecasting in most of introduced STLF methods. But, smart meter data may contain useful features that are not evident in the time domain. Some informative features can be revealed in other feature spaces. To assess this subject, the performance of STLF in time domain is assessed and compared to other domains or feature spaces. LSTM is used for load forecasting in various domains in this work. The assessed domains are time, frequency, cepstrum, spectral centroid, spectral roll-off, spectral flux, energy, time difference, frequency difference, Gabor and collaborative representation (CR). In addition, the performance of LSTM network using hybrid domains is assessed and compared to single domains.

\section{LOAD SEQUENCE TRANSFORMATION}

The load forecasting is done by using a LSTM network that is one of the best deep learning approaches appropriate for sequences and time series. Most of researches use the load sequence in time domain as the input of a predictor. But, the load sequence in time domain may not reveal all useful information related to consumption behavior of customers and variations of electricity demand. In addition, the electrical load sequence has a non-stationary inherence which makes hard prediction. So, it is proposed that extract informative features from the load sequence in other domains in addition to time domain. To this end, different domains are introduced for production of informative features from the load sequence in this section. The main contributions of this work are represented as follows:

1) New features such as collaborative representation are introduced for load data analysis.

2) The performance of LSTM network is assessed in various domains and feature spaces for STLF.

3) Single domains are compared to hybrid ones for STLF through LSTM.

Domain 1: time. $x(n) ; n=0,1, \ldots, N$ is the load sequence in the time domain where $n$ is the time index and $N$ denotes the number of samples.

Domain 2: frequency. The spectral features are extracted from the frequency domain where the coefficients of discrete Fourier transform are defined as follows:

$$
X(f)=\sum_{n=0}^{N-1} x(n) \exp \left(-j \frac{2 \pi}{N} f n\right) ; f=0,1, \ldots, N-1
$$

Domain 3: cepstrum. The cepstral coefficients are obtained from the inverse Fourier transform of logarithm of the absolute (magnitude) of its Fourier transform [25]:

$$
\begin{gathered}
T(n)=\frac{1}{N} \sum_{f=0}^{N-1}\left|\log _{10} X(f)\right| \exp \left(j \frac{2 \pi}{N} f n\right) ; \\
n=0,1, \ldots, N-1
\end{gathered}
$$

Domain 4: spectral centroid. This domain is a measure of spectral shape of load sequence and concentration of load in the frequency domain:

$$
C(i)=\frac{\sum_{k=0}^{m-1}(m-k)|X(i-k)|}{\sum_{k=0}^{m-1}|X(i-k)|+\varepsilon}
$$

where $X(i)$ is $i$ th element of its Fourier transform. $\varepsilon$ is a very small positive constant such as $\varepsilon=10^{-6}$ to avoid becoming zero of denominator. $m$ is a positive integer where $m-1$ previous samples of $X(i)$ are considered for calculating the centroroid value in each point of load sequence.

Domain 5: spectral roll-off. This feature determines $c \%$ (for example $c=80,90$ or 95) of summation of absolute of $m$ Fourier coefficients corresponding to $m$ previous samples of load sequence in the frequency domain [26]. Spectral roll-off reveals the skewness of the spectral shape. It distinguishes where the most of energy is concentrated in the frequency domain:

$$
R(i)=\frac{c}{100} \sum_{k=0}^{m-1}|X(i-k)|
$$

Domain 6: spectral flux. It represents the local changes among successive samples in the frequency domain:

$F(i)=\sum_{k=0}^{m-1}[Q(i-k)-Q(i-k-1)]^{2}$

where $Q(i)$ indicates the absolute value of Fourier coefficient normalized by its maximum value.

Domain 7: energy. Energy of load sequence contained in $m-$ 1 previous samples of load sequence in time domain:

$E(i)=\frac{1}{m} \sum_{k=0}^{m-1}|x(i-k)|^{2}$

Domain 8: time difference. Difference of subsequent samples in time domain is obtained by:

$\operatorname{diff}_{t}(i)=x(i)-x(i-1)$

The differential operator helps to remove the non-stationary behavior of signal. The result is a load sequence with more stationary with respect to the expectation of load.

Domain 9: frequency difference. The differential operator is applied to the load sequence in the frequency domain as follows:

$\operatorname{diff}_{f}(i)=X(i)-X(i-1)$

Domain 10: Gabor features. A Gabor function is a Gaussian function modulated by a sine wave:

$$
G_{\sigma, f_{0}}(i)=g_{\sigma}(i) \exp \left(j 2 \pi f_{0} i\right)
$$

where

$$
g_{\sigma}(i)=\frac{1}{\sqrt{2 \pi \sigma^{2}}} \exp \left(-\frac{i^{2}}{2 \sigma^{2}}\right)
$$

where $\sigma$ is the standard deviation of Gaussian function representing the scale of function and $f_{0}$ indicates the spatial frequency in the complex exponential. The Gabor filter has complex values where the absolute of it is used for determining the contextual features of the load sequence:

$$
\left|G_{\sigma, f_{0}}(i)\right|=\sqrt{R_{\sigma, f_{0}}(i)+I_{\sigma, f_{0}}(i)}
$$

where

$$
\begin{aligned}
& R_{\sigma, f_{0}}(i)=g_{\sigma}(i) \cos \left(2 \pi f_{0} i\right) \\
& I_{\sigma, f_{0}}(i)=g_{\sigma}(i) \sin \left(2 \pi f_{0} i\right)
\end{aligned}
$$

Filtering of the load sequence by using 1D Gabor filter results in characteristics of load in different frequencies and scales in both of spectral and spatial domains. 
Domain 11: collaborative representation $(\boldsymbol{C R})$. The features of CR domain provide neighboring information from the adjacent samples of each point of the load sequence. Let $y$ be the considered sample of load in $i$ th instance, i.e., $y=x(i)$. $m$ previous samples of $y$ compose the $\boldsymbol{x}_{s}$ vector as $\boldsymbol{x}_{s}=$ $[x(i-1), x(i-2), \ldots, x(i-m)]^{T} . y$ can be approximated by previous samples of load sequence through $y=\boldsymbol{\alpha} \boldsymbol{x}_{s}$ where $\boldsymbol{\alpha}$ is the coefficient vector of approximation obtained by:

$\widehat{\boldsymbol{\alpha}}=\arg \min _{\boldsymbol{\alpha}}\left|\boldsymbol{y}-\boldsymbol{\alpha} \boldsymbol{x}_{s}\right|^{2}$

By considering that each sample closer to $y$ has higher similarity to it with a more probability, a larger weight should be assigned to closer samples. To cure this requirement, the objective function in Equation (14) is regularized as follows:

$$
\widehat{\boldsymbol{\alpha}}=\arg \min _{\boldsymbol{\alpha}}\left|y-\boldsymbol{\alpha} \boldsymbol{x}_{s}\right|^{2}+\lambda\left|\boldsymbol{\gamma}_{y} \boldsymbol{\alpha}\right|^{2}
$$

where

$$
\begin{aligned}
& \gamma_{y}=[|y-x(i-1)|,|y-x(i-2)|, \ldots, \mid y- \\
& x(i-m) \mid]^{T}
\end{aligned}
$$

and $\lambda$ is the regularization parameter that controls the relation between two terms. Derivative of the objective function is taken and set to zero. The result will be:

$$
\widehat{\boldsymbol{\alpha}}=\left(\boldsymbol{x}_{s}^{T} \boldsymbol{x}_{s}+\lambda \boldsymbol{\gamma}_{y}^{T} \boldsymbol{\gamma}_{y}\right)^{-1} \boldsymbol{x}_{s}^{T} y=\frac{\boldsymbol{x}_{s}^{T} y}{\left\|\boldsymbol{x}_{s}\right\|^{2}+\lambda\left\|\gamma_{y}\right\|^{2}}
$$

where $\left\|\boldsymbol{x}_{s}\right\|$ is the norm of $\boldsymbol{x}_{s}$ and

$$
\left\|\boldsymbol{\gamma}_{y}\right\|^{2}=\sum_{k=1}^{m}|y-x(i-k)|^{2}
$$

But for samples to be forecasted, $y$ is unknown that has to be predicted. So, instead of $y$, estimate of it denoted by $\hat{y}$ is used. But, what is an appropriate approximation for load sequence in $i$ th instance, i.e., $y=x(i)$ ? The load value in each time instance is close to load value in one previous step, i.e., $x(i-$ $1)$. In addition, the consumed load in each time instance can be close to the consumed load in the same instance of previous year denoted by $x_{p y}(i)$. So, $y=x(i)$ can be approximated by using both of $y=x(i-1)$ and $x_{p y}(i)$ as follows:

$$
\hat{y}=\rho_{a}\left(1-\rho_{b}\right) x(i-1)+\left(1-\rho_{a}\right) \rho_{b} x_{p y}(i)
$$

where $0 \leq \rho_{a} \leq 1$ is the correlation coefficient between $\boldsymbol{x}(i)$ and $\boldsymbol{x}(i-1)$ and $0 \leq \rho_{b} \leq 1$ is the correlation coefficient between $\boldsymbol{x}(i)$ and $\boldsymbol{x}_{p y}(i), \boldsymbol{x}(i)$ is the lagged loads vector of $x(i)$ containing the lagged values of load for $i$ th instance. Corresponding to each point of load sequence, 20 lagged load values are considered that consist of 6 variables related to the dame day from last 3 hours, 7 variables related to the same hour of day before for last 3 hours and 7 variables related to the same hour of previous week for last 3 hours. $\rho_{a}$ and $\rho_{b}$ are calculated by:

$$
\begin{aligned}
& \rho_{a}=\frac{|\boldsymbol{x}(i) \cdot \boldsymbol{x}(i-1)|}{\|\boldsymbol{x}(i)\|\|\boldsymbol{x}(i-1)\|} \\
& \rho_{b}=\frac{\left|\boldsymbol{x}(i) \cdot \boldsymbol{x}_{p y}(i)\right|}{\|\boldsymbol{x}(i)\|\left\|\boldsymbol{x}_{p y}(i)\right\|}
\end{aligned}
$$

where $\boldsymbol{a} \cdot \boldsymbol{b}$ denotes the inner product between vectors $\boldsymbol{a}$ and $\boldsymbol{b}$. If $\boldsymbol{x}(i-1)$ has higher correlation with $\boldsymbol{x}(i), \boldsymbol{x}(i-1)$ has larger weight in approximation of $y=x(i)$ and if $\boldsymbol{x}_{p y}(i)$ has higher correlation with $\boldsymbol{x}(i), \boldsymbol{x}_{p y}(i)$ will have more contribution in approximation of $y=x(i)$. An illustration of load sequence in different domains are shown in Figure 1.

\section{EXPERIMENTAL RESULTS}

The performance of LSTM network is assessed in different domains of smart meter data in this section. The used dataset, assessment measures and settings of structures and free parameters are introduced. Then, the experimental results are reported.

\section{Data, measures and settings}

To assess the performance of the proposed forecasting methods, a consumption load dataset from Ireland is used. This data acquired from Irish social science data archive (ISSDA). It is related to commission for energy regulation (CER) project [27]. The consumed electrical load of 5000
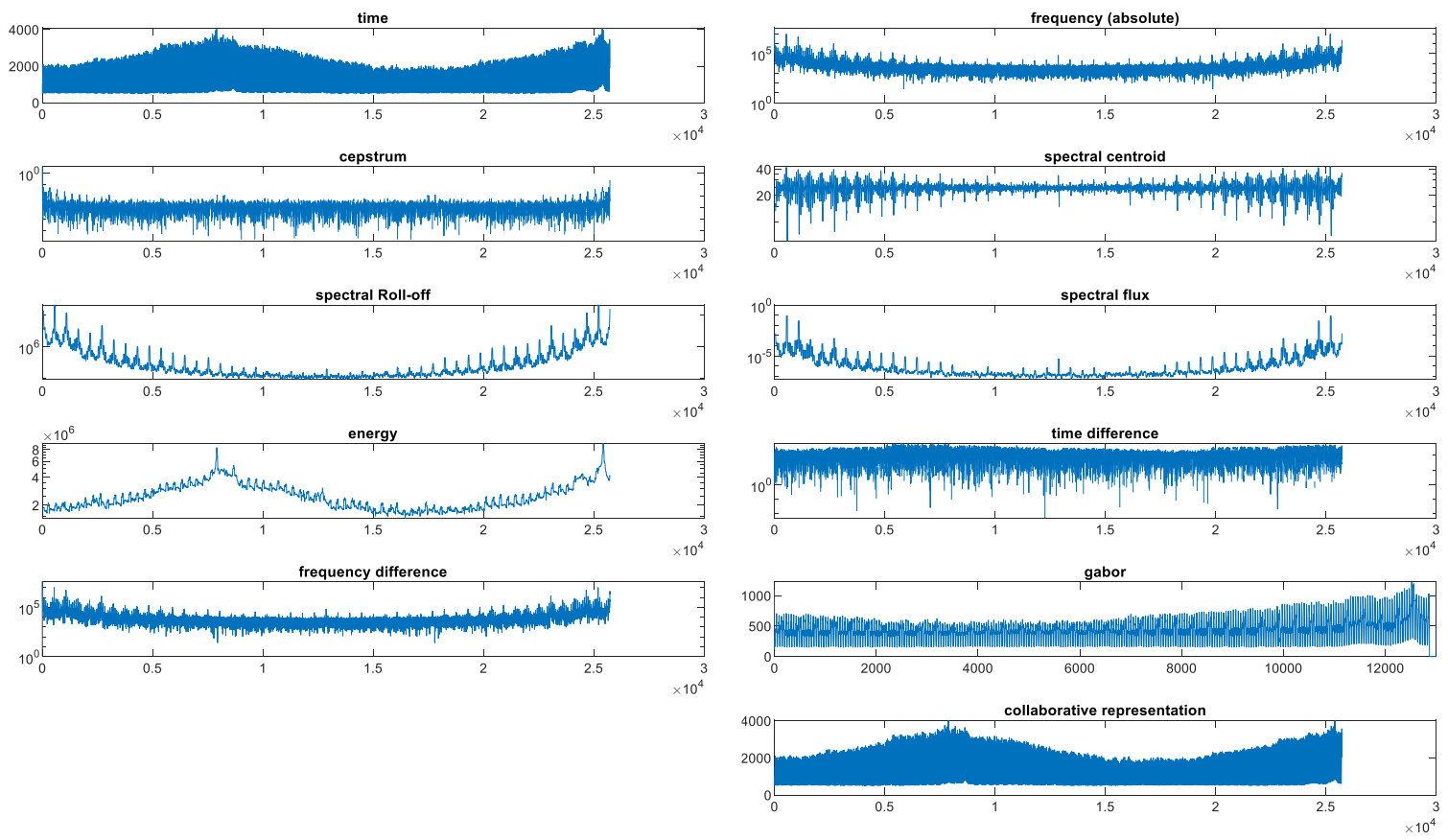

Figure 1. An illustration of load sequence in different domains 
customers (commercial and residential units) from July 2009 to December of 2010 is involved. The residential load is just considered for experiments in this work. Three metrics are used for evaluation of forecasting results: mean absolute percent error (MAPE), mean absolute error (MAE) and mean square error (MSE). MAPE shows the forecasting accuracy, MAE indicates the average error caused by absolute difference between forecasted and actual values and MSE depicts the general deviation between forecasted and actual ones. The best prediction results are corresponding to smallest values of these metrics. Definition of these metrics are as follows:

$$
\begin{aligned}
& M A P E=\frac{1}{N} \sum_{i=1}^{N}\left|\frac{y_{a}(i)-y_{f}(i)}{y_{a}(i)}\right| \\
& M A E=\frac{1}{N} \sum_{i=1}^{N}\left|y_{a}(i)-y_{f}(i)\right| \\
& M S E=\frac{1}{N} \sum_{i=1}^{N}\left|y_{a}(i)-y_{f}(i)\right|^{2}
\end{aligned}
$$

where $y_{f}(i)$ and $y_{a}(i)$ indicate the forecasted and actual values, respectively and $N$ denotes the number of load values to be forecasted. The experiments are done on MATLAB R2018b. $70 \%$ of available data is used for training and the remained data is used for testing. The following structure is considered for LSTM network: 30 hidden units in the LSTM layer, sequence length and mini batch size are equal to $48 \times 7$, the learning rate is equal to 0.001 , and maximum 100 epochs and the 'adam' optimizer are considered. Number of lags, i.e., $m$, in cepstrum, spectral centroid, spectral roll-off, spectral flux and energy domains are experimented with different values of $m=\{2,7,24,48,48 \times 7\}$ and the best forecasting results are obtained by $m=48$. In the CR domain, $m$ is selected as $m=6$ and $\lambda$ is set as $\lambda=10^{-4}$.

\section{Results}

The performance of different introduced domains for STLF is assessed in this section. The main information is contained in the time domain where the original values of load are listed in order of incidence time. So, all domains are used together with the time domain. For example, frequency domain means time domain plus frequency domain; or Gabor domain refers to time domain plus Gabor domain. In addition to single domains, hybrid domains are also assessed. Two combinations of domains are compared. Hybrid1: combination of all domains and hybrid2: combination of domain 1 (time), domain 2 (frequency), domain 3 (cepstrum), domain 4 (spectral centroid), domain 8 (time difference) and domain 9 (frequency difference).

The results of load forecasting in different domains are reported in Table 1. Efficient domains are ranked in terms of MAPE measure as follows: time difference $(0.0056)$-hybrid2 (0.0125), spectral centroid (0.0240), frequency (0.0258), frequency difference $(0.0264)$, cepstrum $(0.0277)$, Gabor (0.0286), spectral roll-off $(0.0315)$, time $(0.0332)$, spectral flux (0.0350), energy (0.0642), hybrid1 (0.0794). The illustration of forecasted values obtained by different single domains together with the actual ones achieved for a $24 \mathrm{~h}$ (containing 48 half hours) are shown in Figure 2. The hybrid results are depicted in Figure 3.

The absolute and relative error curves are also shown in Figures 4-7 for single and hybrid domains.

As seen from the obtained MAPE values, the best forecasting results are achieved by time difference domain. The load sequence is often a non-stationary time series which the statistical behavior of it is not stable during time. The time differencing can degrade the non-stationary nature of load sequence. The non-stable variations that may decrease the learning amount of forecasting network can be removed by differential operator.

The use of the original load values beside the first order time differencing helps LSTM to learn better the variations of load time series. After time difference domain, the spectral
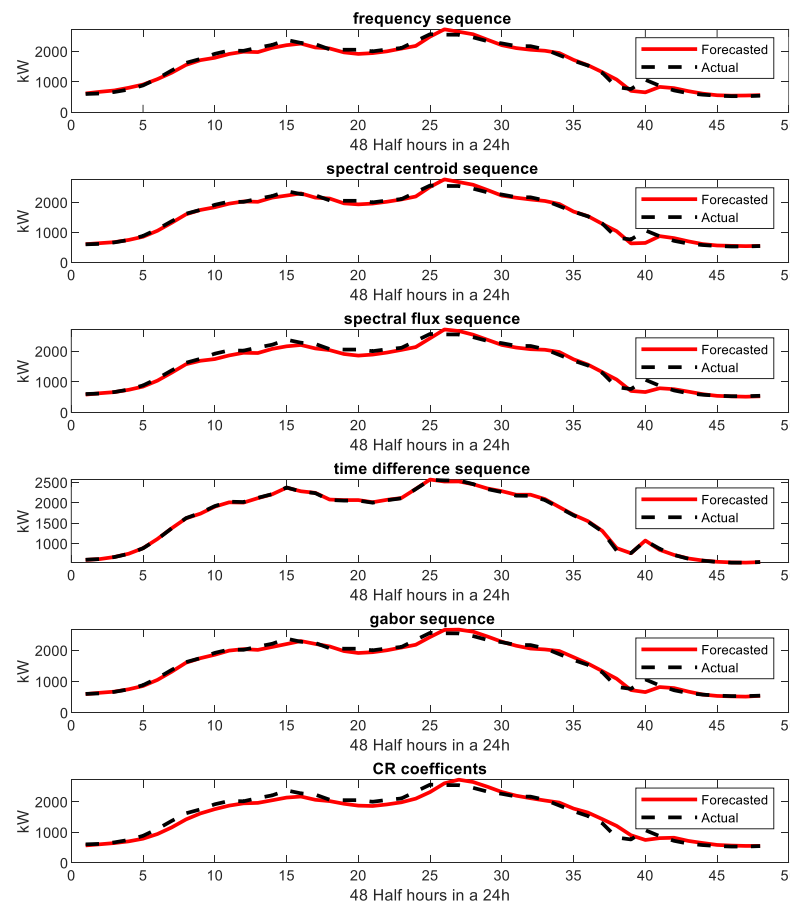

Figure 2. Forecasted load curves obtained by different single domains for a day (48 half hours) 
centroid domain ranks second among the single domains. Spectral centroid is a measure of spectral shape of load sequence in the spectral feature space. Higher value of centroid means that in the high frequencies, there are structures with more energy. This information together with the original values of load time series are effective for learning of non-linear behavior of the electrical load. Among different single domains, the worst result is related to the energy domain that is simply computed by square of load values in the time domain.

In the hybrid1 domain, all 11 domains are combined together. As seen, the worst result is obtained by hybrid 1 domain. This result is due to high redundancy among various features of different domains. In addition, it is found that the use of some features together with other ones may be inconsistent and decreases the learning performance. In contrast, combination of time, frequency, cepstrum, spectral centroid, time difference and frequency difference in hybrid2 domain provides superior results that ranks second after time difference domain. Comparison between hybrid1 and hybrid2 domains concludes that an appropriate combination of domains (feature spaces) can improve the forecasting results while an inappropriate combination degrades the forecasting performance
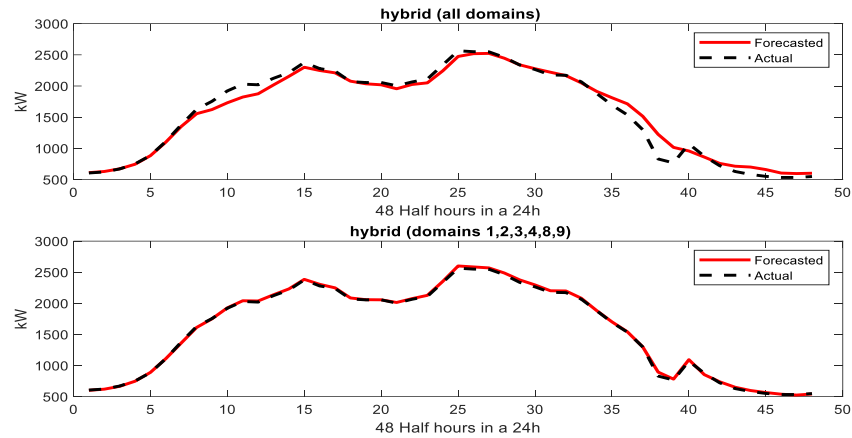

Figure 3. Forecasted load curves obtained by hybrid domains for a day (48 half hours)

TABLE 1. Metric values for comparison of forecasting in different domains

\begin{tabular}{|c|c|c|c|c|c|c|c|c|c|c|c|c|c|}
\hline & $\begin{array}{c}\text { Domain } \\
1\end{array}$ & $\begin{array}{c}\text { Domain } \\
2\end{array}$ & $\begin{array}{c}\text { Domain } \\
3\end{array}$ & $\begin{array}{c}\text { Domain } \\
4\end{array}$ & $\begin{array}{c}\text { Domain } \\
5\end{array}$ & $\begin{array}{c}\text { Domain } \\
6\end{array}$ & $\begin{array}{c}\text { Domain } \\
7\end{array}$ & $\begin{array}{c}\text { Domain } \\
8\end{array}$ & $\begin{array}{c}\text { Domain } \\
9\end{array}$ & $\begin{array}{c}\text { Domain } \\
10\end{array}$ & $\begin{array}{c}\text { Domain } \\
11\end{array}$ & \multicolumn{2}{|c|}{ Several domains } \\
\hline Metric & Time & Frequency & Cepstrum & $\begin{array}{l}\text { Spectral } \\
\text { centroid }\end{array}$ & $\begin{array}{l}\text { Spectral } \\
\text { Roll-off }\end{array}$ & $\begin{array}{l}\text { Spectral } \\
\text { flux }\end{array}$ & Energy & $\begin{array}{c}\text { Time } \\
\text { difference }\end{array}$ & $\begin{array}{l}\text { Frequency } \\
\text { difference }\end{array}$ & Gabor & $\mathrm{CR}$ & $\begin{array}{l}\text { Hybrid1 } \\
\text { (all } \\
\text { domains) }\end{array}$ & $\begin{array}{c}\text { Hybrid2 } \\
\text { (domains } \\
1,2,3,4,8,9 \text { ) }\end{array}$ \\
\hline MAPE & 0.0332 & 0.0258 & 0.0277 & 0.0240 & 0.0315 & 0.0350 & 0.0642 & 0.0056 & 0.0264 & 0.0286 & 0.0388 & 0.0794 & 0.0125 \\
\hline MAE & 45.17 & 38.33 & 43.26 & 34.81 & 48.97 & 53.36 & 89.04 & 9.53 & 40.04 & 43.42 & 56.41 & 107.87 & 18.70 \\
\hline MSE & 3539.78 & 4498.09 & 3741.27 & 2319.17 & 10064.47 & 12141.03 & 18584.89 & 329.61 & 5589.94 & 4323.66 & 5954.96 & 41577.07 & 3203.58 \\
\hline
\end{tabular}
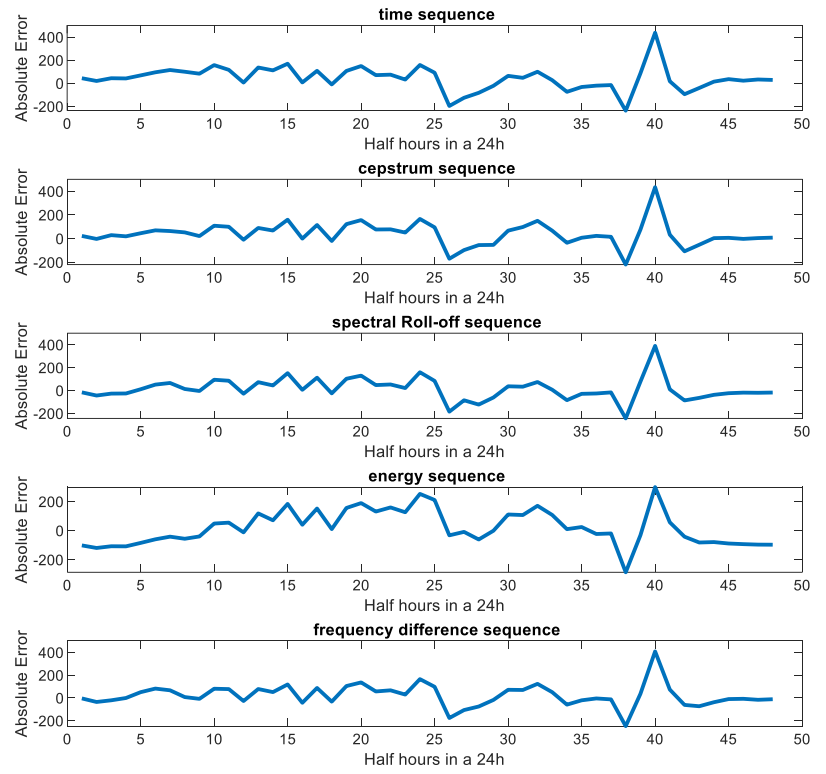

Figure 4. Absolute errors obtained by different single domains for a day (48 half hours)
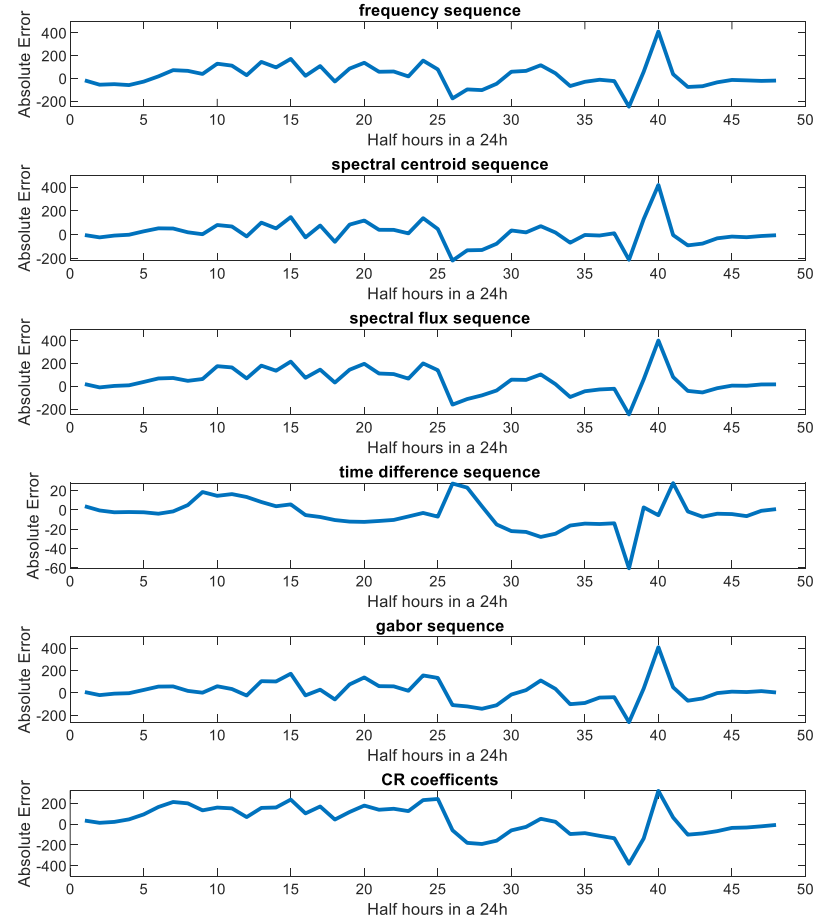

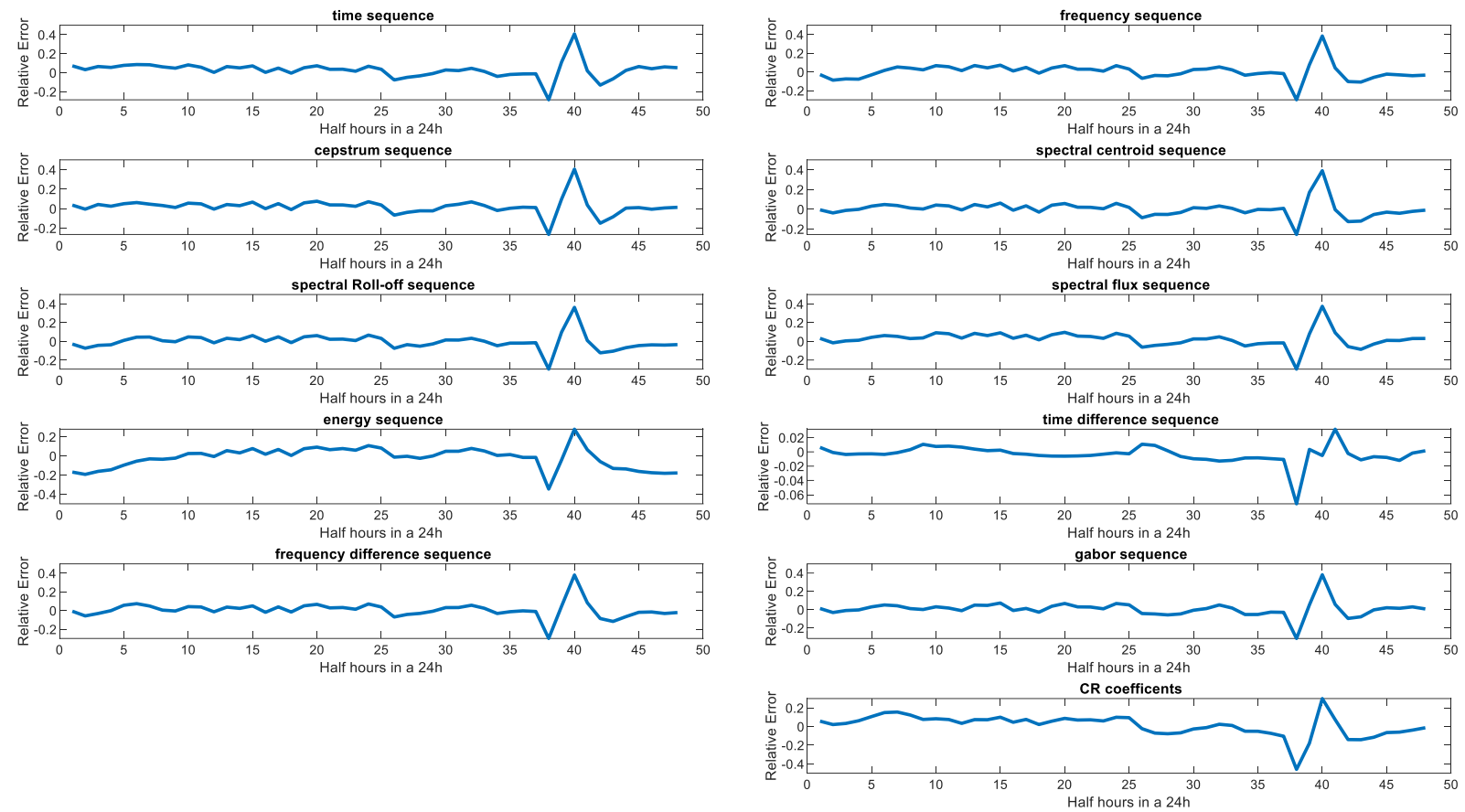

Figure 5. Relative errors obtained by different single domains for a day (48 half hours)
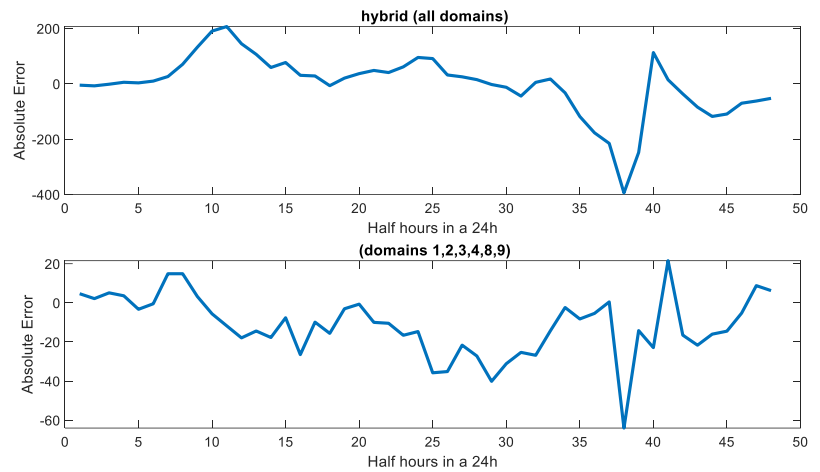

Figure 6. Absolute errors obtained by hybrid domains for a day (48 half hours)
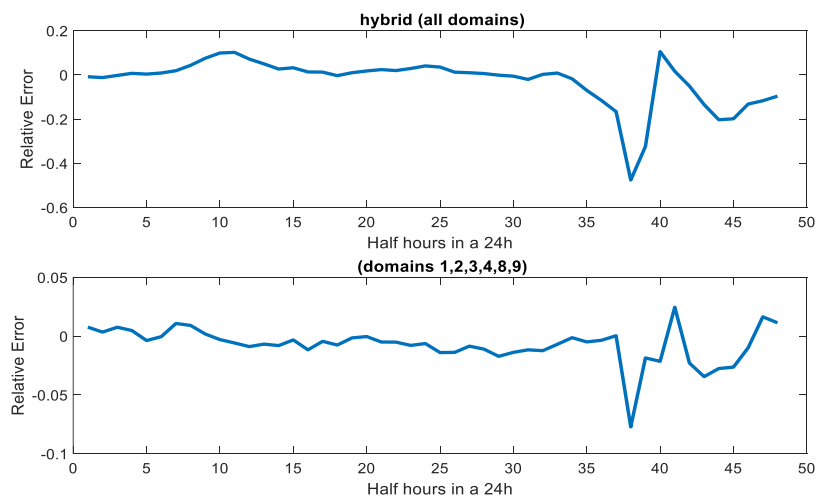

Figure 7. Relative errors obtained by hybrid domains for a day (48 half hours)

\section{CONCLUSION}

Electricity demand forecasting in various domains of time, frequency, cepstrum, spectral centroid, spectral roll-off, spectral flux, energy, time difference, frequency difference, Gabor and collaborative representation is investigated in this paper. Generally, selection of a single or hybrid domain, i.e., an appropriate feature space of load sequence, can provide improvement in load forecasting. The time difference domain not only provides a rich source of information about electrical load variations but also decreases the non-stationary behavior of load sequence. So, integration of it with original time series significantly improves the electrical load forecasting. Other domains such as frequency, cepstrum, spectral centroid and frequency difference cannot be efficient lonely. But, an appropriate combination of them can improve the forecasting result.

\section{REFERENCES}

1. Sahay, K.B. and Rana, V., 2015. One hour ahead load forecast of PJM electricity market \& UPPCL. In 2015 International Conference on Energy Economics and Environment, IEEE, pp. 1-6.

2. Ali, M., Adnan, M. and Tariq, M., 2019. Optimum control strategies for short term load forecasting in smart grids. International Journal of Electrical Power \& Energy Systems, 113, pp.792-806.

3. Torabi, M., Hashemi, S., Saybani, M.R., Shamshirband, S. and Mosavi, A., 2019. A Hybrid clustering and classification technique for forecasting short-term energy consumption. Environmental Progress \& Sustainable Energy, 38(1), pp.66-76.

4. Quilumba, F.L., Lee, W.J., Huang, H., Wang, D.Y. and Szabados, R.L., 2014. Using smart meter data to improve the accuracy of intraday load forecasting considering customer behavior similarities. IEEE Transactions on Smart Grid, 6(2), pp.911-918.

5. Du, X., Bai, J. and Fan, Q., 2011. Study of the medium-long load forecasting based on the identical dimension addition grey model. In 2011 Second International Conference on Mechanic Automation and Control Engineering, IEEE, pp. 700-703.

6. Carmo, D.L., Souza, R.C. and Barbosa, C.R.H., 2020. Partial decomposition approach to generate load curve forecasting scenarios. International Journal of Electrical Power \& Energy Systems, 115, 
p.105436. https://doi.org/10.1016/j.ijepes.2019.105436.

7. Kumar, S., Mishra, S. and Gupta, S., 2016, February. Short term load forecasting using ANN and multiple linear regression. In 2016 Second International Conference on Computational Intelligence \& Communication Technology, IEEE, pp. 184-186.

8. Liu, S.T., Huang, S.R. and Hsien, T.Y., 2005. Optimal identification of self-reunion multiple regression (SRMR) model based on regression function for short-term load forecasting. In 2005 International Power Engineering Conference, IEEE, pp. 1-46.

9. Wei, W., Yin, Q., Bai, Y., Bao, H., Fang, C. and Yang, J., 2013. Regional load forecasting method based on the S-curve power business expansion. In 2013 IEEE International Conference of IEEE Region 10, IEEE, pp. 1-4.

10. Matsumoto, T., Nakajima, Y., Saito, M., Sugi, J. and Hamagishi, H., 2001. Reconstructions and predictions of nonlinear dynamical systems: A hierarchical Bayesian approach. IEEE transactions on signal processing, 49(9), pp.2138-2155.

11. Taylor, J.W. and McSharry, P.E., 2007. Short-term load forecasting methods: An evaluation based on european data. IEEE Transactions on Power Systems, 22(4), pp.2213-2219.

12. El Desouky, A.A. and El Kateb, M.M., 2000. Hybrid adaptive techniques for electric-load forecast using ANN and ARIMA. IEE Proceedings-Generation, Transmission and Distribution, 147(4), pp.213-217.

13. Vrablecová, P., Ezzeddine, A.B., Rozinajová, V., Šárik, S. and Sangaiah, A.K., 2018. Smart grid load forecasting using online support vector regression. Computers \& Electrical Engineering, 65, pp.102117.

14. Jiang, H., Zhang, Y., Muljadi, E., Zhang, J.J. and Gao, D.W., 2016. A short-term and high-resolution distribution system load forecasting approach using support vector regression with hybrid parameters optimization. IEEE Transactions on Smart Grid, 9(4), 3341-3350.

15. Perfumo, C., Braslavsky, J.H. and Ward, J.K., 2014. Model-based estimation of energy savings in load control events for thermostatically controlled loads. IEEE Transactions on Smart Grid, 5(3), pp.14101420 .

16. Srinivasan, D., Tan, S.S., Chang, C.S. and Chan, E.K., 1998. Practical implementation of a hybrid fuzzy neural network for one-day-ahead load forecasting. IEE Proceedings-Generation, Transmission and Distribution, 145(6), pp.687-692.
17. Mota, L.T.M., Mota, A.A. and Morelato, A., 2007. Load behaviour prediction under blackout conditions using a fuzzy expert system. IET Generation, Transmission \& Distribution, 1(3), pp.379-387.

18. Nose-Filho, K., Lotufo, A.D.P. and Minussi, C.R., 2011. Short-term multinodal load forecasting using a modified general regression neural network. IEEE Transactions on Power Delivery, 26(4), pp.2862-2869.

19. Quan, H., Srinivasan, D. and Khosravi, A., 2013. Short-term load and wind power forecasting using neural network-based prediction intervals. IEEE Transactions on Neural Networks and Learning Systems, 25(2), pp.303-315.

20. Samet, H., Reisi, M. and Marzbani, F., 2019. Evaluation of neural network-based methodologies for wind speed forecasting. Computers \& Electrical Engineering, 78, pp.356-372.

21. Bebis, G. and Georgiopoulos, M., 1994. Feed-forward neural networks. IEEE Potentials, 13(4), pp.27-31.

22. Shao, H., 2008. Delay-dependent stability for recurrent neural networks with time-varying delays. IEEE Transactions on Neural networks, 19(9), pp.1647-1651.

23. Wang, S., Wang, X., Wang, S. and Wang, D., 2019. Bi-directional long short-term memory method based on attention mechanism and rolling update for short-term load forecasting. International Journal of Electrical Power \& Energy Systems, 109, pp.470-479.

24. Alawe, I., Ksentini, A., Hadjadj-Aoul, Y. and Bertin, P., 2018 Improving traffic forecasting for $5 \mathrm{G}$ core network scalability: A Machine Learning approach. IEEE Network, 32(6), pp.42-49.

25. Kaur, A. and Singh, A., 2016. Power-Normalized Cepstral Coefficients (PNCC) for Punjabi Automatic Speech Recognition using phone based modelling in HTK. In 2016 2nd International Conference on Applied and Theoretical Computing and Communication Technology, IEEE, pp. 372-375.

26. Theodoridis, S. and Koutroumbas, K., 2010. Pattern recognition \& Matlab intro. Academic Press, Inc.

27. Commission for Energy Regulation (CER) Smart Metering Project, 2009-2010 Dataset, Electricity Customer Behaviour Trial, 1st Edition. Irish Social Science Data Archive. SN: 0012-00. www.ucd.ie/issda/CER-electricity, 2012. 